All letters are subject to editing and may be shortened. Letters should be sent to the BJGP office by e-mail in the first instance, addressed to

journal@rcgp.org.uk (please include your postal address). Alternatively, they may be sent by post as an MS Word or plain text version on CD or DVD. We regret that we cannot notify authors regarding publication. Letters not published in the Journal may be posted online on our Discussion Forum. For instructions please visit: http://www.rcgp.org.uk/bjgp-discuss

\section{OTC cough remedies}

Your editorial by Smith, et al ${ }^{1}$ made very interesting reading. However, one feature perhaps overlooked was GPs prescribing or recommending such OTC cough medicines solely for the purpose of avoiding the dreaded alternative of the inappropriate antibiotic. Doubtless, we must continue to educate our patients and also endeavour to promote appropriate help-seeking behaviour. Unfortunately, there will probably always be the small cohort of patients/parents/guardians whereby the consultation must end with 'the prescription'. For many of us, this may well continue to be the tried and trusted OTC preparation of our choosing, despite current evidence. We need more such articles which challenge our daily working habits in an evidence-based manner. Well done.

\section{John Peters}

Littlepace Medical Centre, Littlepace Shopping Mall, Clonee, Dublin 15. E-mail: john.peters@bms.com

\section{REFERENCE}

1. Smith SM, Henman M, Schroeder K, and Fahey T. Over-the-counter cough medicines in children: neither safe or efficacious? Br J Gen Pract 2008; 58(556): 757-758.

DOI: 10.3399/bjgp09X395148

\section{Authors' response}

Thank you for your response to our editorial. We appreciate and often share your dilemma regarding some patients' expectations that they will receive something concrete at the end of a consultation and some of which are potentially more harmful than others. We have suggested, the 'traditional' honeybased preparations could be recommended and in addition, relief of fever particularly at night, could substantially reduce discomfort.

However, we would advise strongly against using antihistamines and decongestants, singly or in combination, because of their potential for harm as much as for the existing evidence that implicates them. We would also advise against the use of antitussives although the evidence is not as clear cut, and prescribers with extensive experience of these agents will quite appropriately rely on their own judgement. Of the nonprescription ingredients that remain, guaiphenesin is probably the safest along with mucolytics. While the evidence for the effectiveness of these agents is limited, their potential for harm given their mechanisms of action is also very limited.

Perhaps the provision of an alternative such as a leaflet would satisfy these patients and studies of these types of interventions are ongoing. Such patient information leaflets should incorporate the following messages:

- check the suitability of the product with a prescriber or pharmacist;

- do not use products intended for older children or for adults, even in reduced dosage;

- ensure child-minders are instructed carefully about product identity, doses, and frequency of administration; and

- ensure that the child is regularly offered fluids since the idea of fever producing dehydration is often overlooked by anxious parents.
In addition, GPs need to be aware of the risk of recommending these preparations in 'at risk' families/groups who may use them as sedatives rather than for symptom relief. Finally, as we mentioned, parents need to be fully aware of the likely duration of cough symptoms particularly as patient information leaflets for non-prescription preparations recommend seeking advice early rather than late, which tends to reinforce the idea that persistent symptoms are unexpected. So, parents can be reassured that unless there is a deterioration in the child's condition, supportive care will suffice.

\section{Susan Smith}

Department of Public Health and Primary Care, Trinity College Centre for Health Sciences, AMNCH, Tallaght, Dublin 24. E-mail: susmith@tcd.ie

\section{Martin Henman}

Centre for the Practice of Pharmacy, The School of Pharmacy and Pharmaceutical Science, Trinity College, Dublin.

\section{Knut Schroeder}

Academic Unit of Primary Health Care, University of Bristol, Bristol.

\section{Tom Fahey}

Department of General Practice, Royal College of Surgeons in Ireland, Dublin.

DOI: 10.3399/bjgp09X395157 